results of their interference therefore produces essentially straight-line or particle-like propagation. In other words the apparent contradiction between particle and wave concepts now disappears and for the same reason as it did in the particle-wave controversy of a hundred fifty years ago, between Newton and Huygens.

In closing my tribute to Einstein I wish to say that much as I honor him for his immense contributions to physics, his greatest qualities lie in the field of character and morals. I worked with him for some years on a committee of the League of Nations and I also saw much of him in the two winters which he spent with us at the California Institute of Technology, and I came to admire him most for his extraordinary open-mindedness, his modesty, his honesty, and his complete readiness to admit that he had been wrong and to change his position entirely in the light of new conditions. His two-page statement found in a small pamphlet entitled "My Faith," printed and distributed by the American Weekly (New York, 1948) reveals a greatness of soul and keenness of intelligence and understanding rarely found in the history of mankind.

\title{
L'Oeuvre d'Einstein et la Dualité des Ondes et des Corpuscules*
}

\author{
L. DE BRoglie \\ Institut Henri Poincaré, Sorbonne, Paris
}

\begin{abstract}
M Albert Einstein dont tout le monde savant 1. célèbre aujourd'hui le soixante-dixième anniversaire a accompli une oeuvre scientifique d'une immense portée: en dehors de ces importants travaux sur la théorie des fluctuations et sur le mouvement Brownien, il a créé entièrement la théorie de la Relativité qui a tenu à juste titre une si grande place dans la Physique contemporaine et il a, à plusieurs reprises, apporté des contributions essentielles au développement de la théorie des Quanta. C'est lui en particulier qui a le premier souligné la dualité d'aspect onde-corpuscule dans le cas de la lumière et qui en a très finement analysé quelques unes des conséquences les plus importantes. Nous voudrions montrer dans les pages qui suivent le lien qui existe entre les deux parties essentielles de l'oeuvre d'Einstein; ce n'est pas par hasard que le créateur de la théorie de la Relativité a été aussi le précurseur de la Mécanique ondulatoire et des théories quantiques actuelles.

Le point de départ de la théorie de la Relativité restreinte a été l'invariance de l'equation de propagation des ondes lumineuses pour tous les observateurs galiléens, invariance qu'on peut considérer comme prouvée par la célèbre expérience de Michelson et d'autres expériences analogues. L'invariance en question entraine que l'on doit établir entre les coordonnées d'espace et de temps utilisées par divers observateurs galiléens des relations linéaires qui constituent les fameuses formules de Lorentz. Analysant avec une géniale profondeur les conditions de mesure des longueurs et des durées, $\mathbf{M}$. Einstein a interprété les formules de Lorentz en
\end{abstract}

* Editor's note: Because of the nature of this special issue, we are departing from our general practice of publishing only in the English language. admettant le caractère relatif de l'espace et du temps et en abandonnant ainsi la vieille conception newtonienne du "temps universel." Il a ainsi donné à la contraction de Lorentz et au ralentissement des horloges par le mouvement, qui sont des conséquences simples de la transformation de Lorentz, le caractère d'apparences observables. Puis il a montré que ces conceptions nouvelles conduisaient à une nouvelle cinématique reposant essentiellement sur de nouvelles formules de composition des vitesses différentes des formules classiques.

Cette réforme de la Cinématique devait inévitablement, Paul Langevin l'a montré plus tard, entrainer une réforme de la Dynamique. Il était d'ailleurs naturel d'admettre que les phénomènes mécaniques devaient être invariants pour le même groupe de transformation que les phénomènes électromagnétiques. Jusque là, on avait admis que les phénomènes mécaniques étaient invariants pour le groupe de Galilée, mais ce fait n'était vérifié qu'avec la précision assez limitée des mesures mécaniques; l'expérience de Michelson venant apporter, avec la très haute précision des mesures interférométriques, la preuve que les phénomènes électromagnétiques sont invariants pour le groupe de Lorentz, Einstein a été naturellement amené à penser qu'il fallait transformer les équations de la Dynamique de façon à les rendre invariantes pour le groupe de Lorentz, tout en conservant la validité approximative des équations anciennes dans le domaine des mouvements usuels à grande échelle où elles se sont montrées bien adaptées à la description des faits. Ainsi s'est développée la Dynamique relativiste qui ne diffère de la Dynamique ancienne que si les corps en mouvement sont animés de vitesses voisines de la vitesse $c$ de la lumière dans le vide. L'une des plus remarquables conséquences de la Dynamique nouvelle a 
été le principe de l'inertie de l'énergie qu'Einstein a illustré par de nombreux exemples.

Si nous réfléchissons au résultat final de ces magnifiques recherches, nous aperçevons qu'elles conduisaient à un rapprochement sensible entre la théorie des ondes et la théorie des corpuscules. La transformation de Lorentz pour laquelle l'équation des ondes électromagnétiques est invariante provient ainsi de la théorie ondulatoire et, en modifiant la Dynamique des corpuscules de façon à la rendre invariante pour cette même transformation de Lorentz, on crée implicitement une sorte de solidarité entre les ondes et les corpuscules. Einstein le savait bien: aussi n'est-il pas étonnant de constater que, dans le courant de la même année 1905, on voit germer dans son puissant cerveau les conceptions fondamentales de la Relativité et l'hypothèse des "Quanta de lumière." Sans doute, quand Einstein a admis que dans les rayonnements électromagnétiques, il existe des quanta de lumière, des photons comme nous disons aujourd'hui, il avait surtout en vue d'interprèter les lois de l'effet photoélectrique et on sait avec quelle merveilleuse simplicité il y parvint. Mais il fût certainement encouragé dans cette voie par toute une série de concordances que seules les idées relativistes permettaient de mettre en lumière. Nous allons en rappeler sommairement quelques unes.

Tout d'abord, Einstein pour rendre compte des lois de l'effet photoélectrique posait en accord avec les idées de Planck sur les Quanta la relation

$$
W=h \nu,
$$

qui définit l'énergie $W$ d'un photon en fonction de la fréquence $\nu$ de l'onde qu'il accompagne. De là on peut déduire, Einstein l'a fait de diverses façons, que la quantité de mouvement $g$ du photon qui est naturellement dirigée dans le sens de propagation de l'onde a pour grandeur

$$
g=h \nu / c=W / c .
$$

Or ce résultat est une conséquence immédiate de la Dynamique relativiste qui établit entre l'énergie $W$ et la quantité de mouvement $g$ d'un corpuscule quelconque la relation générale

$$
g=\left(W / c^{2}\right) v,
$$

où $v$ est la vitesse. Pour le photon, on doit supposer $v=c$ et tenir compte de la relation (1), ce qui donne immédiatement la formule (2).

La relation ainsi établie entre l'énergie et la quantité de mouvement d'un photon est précisément celle qui existe d'après la théorie électromagnétique entre la quantité de mouvement et l'énergie d'une onde électromagnétique, comme on le sait depuis les travaux de Max Abraham et d'Henri Poincaré. Si on appliquait au photon la mécanique classique on obtiendrait un résultat tout différent puisqu'alors on aurait pour le photon $g=(2 m W)^{\frac{1}{2}}$ et il serait impossible de mettre cette relation en accord avec celle fournie par la théorie électromagnétique.
On retrouve des conclusions analogues quand on étudie la pression de radiation. Comme Planck l'avait indiqué dans son livre "Wärmestrahlung," si l'on applique au photon les formules de la Mécanique newtonienne, on trouve aisément pour la pression exercée par une radiation sur un miroir parfait qui la réfléchit une valeur qui se trouve être exactement le double de celle prévue par la théorie électromagnétique; cette divergence disparait si l'on fait usage pour le photon de la dynamique einsteinienne relativiste, car on retrouve alors exactement la valeur de la pression de radiation fournie par la théorie électromagnétique et vérifiée par l'expérience.

On voit ainsi qu'il aurait été impossible d'obtenir une théorie des photons satisfaisante si l'on n'avait connu que la Mécanique classique. Mais la Mécanique relativiste, évidemment seule applicable à une particule se déplaçant avec une vitesse qui tend vers celle de la lumière, permet d'obtenir des résultats conciliables avec ceux de la théorie électromagnétique. On voit ainsi comment la théorie de la Relativité a rendu possible une synthèse des points de vue ondulatoire et corpusculaire en ce qui concerne la lumière et ceci montre bien le lien profond qui existe entre les deux grandes découvertes effectuées par Albert Einstein.

Les formules de la Dynamique relativiste appliquées au photon permettent aussi de retrouver complètement les diverses sortes d'effets Doppler et l'aberration:elles conduisent encore exactement aux mêmes résultats que la théorie électromagnétique sous sa forme relativiste.

Dans un autre domaine, l'application de la Dynamique relativiste au photon conduit aussi à un résultat remarquable. L'hypothèse des quanta de lumière conduit naturellement à considérer le rayonnement noir comme un gaz de photons qui, à la température absolue $T$, est en équilibre avec la matière comme une vapeur avec sa phase condensée. En développant cette idée dans le cadre de la Mécanique statistique classique de Boltzmann-Gibbs, on trouve pour la loi de répartition spectrale des énergies dans le rayonnement noir la forme proposée par Wien, forme qui représente bien les résultats expérimentaux pour les grandes valeurs du quotient $\nu / T$, alors que la formule de Rayleigh-Jeans déduite des idées classiques concorde avec les faits pour les petites valeurs du même quotient. La loi exacte est celle de Planck qui représente exactement pour toutes les valeurs de $\nu / T$ la répartition spectrale en question; la loi de Planck admet la loi de Rayleigh-Jeans et celle de Wien comme formes approximatives limites respectivement pour les petites valeurs et pour les grandes valeurs de $\nu / T$. Ici donc l'application de la Dynamique relativiste au photon conduit à un résultat qui n'est que partiellement exact. Nous savons aujourd'hui que ce demi-échec provient de l'insuffisance de la Mécanique statistique de Boltzmann-Gibbs. Dès 1910, Einstein consacrait de profondes études à l'origine de la loi de Planck en analysant les fluctuations dans le rayonnement noir. Plus tard, en Janvier 1925, ayant eu connaissance d'un 
travail du physicien Hindou Bose ainsi que des premières recherches de l'auteur du présent article sur la Mécanique ondulatoire, Einstein montrait que, pour obtenir la loi de Planck, il fallait modifier les méthodes statistiques classiques de façon à tenir compte de l'indiscernabilité des particules de même nature; il fondait ainsi la première des statistiques quantiques, celle qui a gardé le nom de statistique de Bose-Einstein. Peu de temps après, on s'est rendu compte que seules certaines catégories de particules (dont font partie les photons) obéissent à cette statistique; ce sont les particules qui ne sont pas soumises au principe d'exclusion de Pauli. Par contre, les particules soumises au principe de Pauli obéissent à une autre statistique quantique connue aujourd'hui sous le nom de statistique de Fermi-Dirac. Tout ceci est bien connu, mais il n'est pas inutile de rappeler la grande contribution qu'Einstein a apportée à l'établissement de ces théories nouvelles.

Le rapprochement entre la théorie des ondes et la Mécanique corpusculaire effectué par l'oeuvre d'Einstein a joué un grand rôle dans l'éclosion de la Mécanique ondulatoire. D'une part, le succès de la théorie des photons amenait à se demander si, pour les particules qu'elles soient particules de matière ou particules de lumière, il n'y a pas lieu de considérer un aspect ondulatoire et un aspect corpusculaire. D'autre part l'analogie, bien connue depuis Hamilton, entre la Mécanique analytique et l'optique géométrique conduit à des idées analogues. Le procédé qui, le premier, a permis de préciser ces intuitions encore vagues a été d'inspiration essentiellement relativiste. Dans le système propre d'un corpuscule en mouvement rectiligne et uniforme, l'énergie $W_{0}$ est égale à $m_{0} c^{2}$ et la quantité de mouvement $g_{0}$ est nulle. Si dans ce système, nous cherchons à associer à la présence du corpuscule un élément périodique nous sommes amenés tout naturellement à supposer qu'il a la forme d'une onde stationnaire dont la représentation complexe sera $\psi=a \mathrm{e}^{2 \pi i \nu_{0} t_{0}}$. La relation établie par Einstein entre l'énergie et la fréquence dans la théorie du photon nous conduit à supposer que l'on a $W_{0}=h \nu_{0}$. Si maintenant on se place dans un système Galiléen où le corpuscule a la vitesse $\beta c$ dans la direction $o x$, l'énergie $W$ sera égale à $\left[m_{0} c^{2} /\left(1-\beta^{2}\right)^{\frac{1}{2}}\right]$ et la quantité de mouvement à $\left[m_{0} \beta c /\left(1-\beta^{2}\right)^{\frac{1}{2}}\right]$. Quant à l'élément periodique $\psi$, il prendra la forme

avec

$$
\begin{gathered}
\psi=a \exp 2 \pi i \nu[t-(x / V)], \\
\nu=\nu_{0} /\left(1-\beta^{2}\right)^{\frac{1}{2}}, \quad V=c / \beta=c^{2} / v,
\end{gathered}
$$

comme on le voit immédiatement en remplaçant dans l'expression primitive de $\psi$ la valeur de $t_{0}$ en fonction de $t$ et de $x$, donnée par la transformation de Lorentz. L'élément $\psi$ possède donc dans ce système de référence l'aspect d'une onde se propageant dans la direction $o x \mathrm{du}$ mouvement et possèdant la fréquence $\nu$ et la longueur d'onde $\lambda$ données par les formules

$$
\nu=W / h, \quad \lambda=V / \nu=h / g .
$$

L'expression de $\lambda$ a pu être vérifiée pour la première fois lorsque MM. Davisson et Germer sont parvenus en 1927 à obtenir la diffraction des électrons par les cristaux. L'expression de $\nu$ n'est pas vérifiable directement, mais elle est la seule qui soit conforme aux règles de la variance relativiste.

La seconde formule (4) montre que la vitesse de phase $V$ varie en raison inverse de la vitesse corpusculaire $v$. Ceci rend possible l'identification du principe de moindre action de Maupertuis appliqué au corpuscule au principe de Fermat appliqué à l'onde $\psi$ qui s'écrive respectivement

$$
\delta \int_{A}^{B} \frac{d l}{\lambda}=\nu \delta \int_{A}^{B} \frac{d l}{V}=0 \quad \text { et } \delta \int_{A}^{B} g d s=0 .
$$

Cette identification résulte immédiatement des formules (4) et (5). On établit ainsi une coincidence entre l'optique géométrique de l'onde et le mouvement du corpuscule qui peut être généralisé au cas du mouvement dans un champ. Comme l'optique géométrique n'est du point de vue de l'optique ondulatoire qu'une approximation, on doit penser qu'il en est de même de la Mécanique ancienne (même sous sa forme relativiste). C'est pourquoi j'écrivais, dès le début de mes recherches sur ce sujet, qu'il fallait tenter de constituer une mécanique nouvelle qui soit par rapport à l'ancienne "ce qu'est l'optique ondulatoire par rapport à l'optique géométrique." On sait comment la Mécanique ondulatoire s'est effectivement développée en ce sens grâce aux brillants travaux de M. Schrödinger. Je n'insisterai pas sur l'immense développement qu'a pris depuis la Mécanique ondulatoire, me bornant à rappeler le rôle qu'ont encore joué les considérations d'invariance relativiste dans la naissance de la belle théorie de l'électron magnétique de M. Dirac.

Ce qu'il importe de souligner ici, c'est l'influence décisive qu'ont eue les idées d'Einstein dans le développement de la Physique atomique contemporaine. Lorsqu'en 1905 il a presque simultanément découvert le principe de Relativité et élargi la théorie des quanta par l'hypothèse des quanta de lumière, il a apporté aux physiciens des idées essentielles sans lesquelles ils n'auraient pu progresser dans la voie où ils étaient engagés. Et c'est l'une des raisons pour lesquelles son oeuvre capitale domine toute la période contemporaine de l'histoire des Sciences. 